\title{
Thorax annual report: 1 October 2003 to 30 September 2004
}

\section{J A Wedzicha, S L Johnston, D M Mitchell}

$\mathrm{T}$ he past year-and the second year of our editorship-has been a very successful one for Thorax. ${ }^{12}$ We have continued to receive high quality papers for publication and our impact factor for 2003 now stands at 4.188 . $^{3}$ This means that Thorax is now the second highest ranked of the traditional respiratory journals in terms of impact factor, behind the American Journal of Respiratory and Critical Care Medicine, and has now overtaken the American Journal of Respiratory Cell and Molecular Biology.

The journal impact factor for 2003 is calculated as the number of citations in 2003 to papers published in Thorax in 2001 and 2002. The changes in impact factor for Thorax from 1996 to 2003 and a comparison with the impact factors for the other main respiratory journals are shown in fig $1 .^{3-5}$ The impact factor of a journal depends on the number of high quality papers that it publishes and this is influenced by the rigour of the peer review in the journal. ${ }^{6}$ For some time now, in addition to regular peer review in Thorax, all potentially acceptable papers have been subject to statistical review and this has had a positive effect on the quality of the papers we have published. The impact factor is also affected by the number of articles published in a journal and this makes up the denominator of the impact factor equation. Some specialist journals have reduced-often quite dramaticallythe number of papers they publish. However, the number of articles (excluding editorials and letters) published in Thorax has remained relatively constant, with 183 articles in 2001, 210 in 2002, and 198 in 2003. The improvement in the impact factor therefore reflects the increased number of citations in the medical literature to Thorax papers.

The number of submissions to Thorax has increased further compared with last year; from October 2002 to September 2003 the number of submissions was 1260 and this has now risen to 1597 between October 2003 and September 2004. The number of original research articles submitted to the journal has also increased from 860 last year to 1028. Table 1 shows the number of submissions to Thorax by article type.

We have also seen an increase in submissions from outside the UK, with an especially sharp rise in submissions from Europe and continuing increases from North America, Canada, Asia Pacific, and Australasia. Table 2 shows the geographical distribution of submissions to Thorax.

The time to the first decision on a paper has reduced to a median of 27.5 days. However, the increased number of submissions means the acceptance rate for original research papers now stands at only $12 \%$, and $6 \%$ for case reports.

This year we have published some important original papers and useful management guidelines for common conditions such as radiologically guided lung biopsy in November $2003^{78}$ and the NICE COPD guidelines in March $2004,{ }^{9}{ }^{10}$ with a description of the peer review process that was followed during the development of the guidelines. ${ }^{11} \mathrm{~A}$ number of review series on common and important issues in respiratory medicine have been published in the journal. We have completed the popular review series on lung cancer ${ }^{12-14}$ and started new review series on cough, ${ }^{15-22}$ sleep disordered breathing, ${ }^{23-28}$ and $\alpha_{1}$ antitrypsin deficiency. ${ }^{29-34}$ Sadly, Moran Campbell died this year and John Gibson-in a truly splendid article in the journal-described his remarkable achievements as a clinician, researcher, and teacher. ${ }^{35}$ We also published a report on the British Thoracic Society Winter meeting held in London in December 2003. ${ }^{36}$

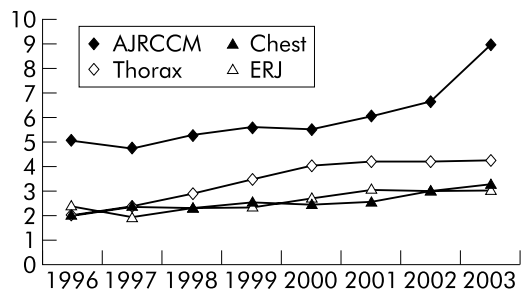

Figure 1 Impact factors for main respiratory journals.
We have continued a number of features aimed at improving the educational value of Thorax. Every month we produce an "Airwaves" section at the front of the journal with short paragraphs highlighting the key messages of some of the papers in the issue. The Lung Alerts, in which we publish short reviews of respiratory papers in other general and specialist journals, are very popular. ${ }^{37}$ We have had an excellent response to our call to younger Thorax readers for help with this feature, and we are very grateful to all the contributors who have made it such a success this year. We would like to thank Angshu Bhowmik and John Hurst for organising the Lung Alerts each month and keeping strictly to all the journal publication deadlines. The Thorax website (www.thoraxjnl.com) has proved very popular and our 10 most frequently read articles online in 2003 had a total of 104602 accesses as either full text, PDF versions, or as abstracts. ${ }^{38-47}$

In November 2003 we launched a new monthly educational series called "Images in Thorax" in which we publish a radiological image or a pathological section with a short explanatory note and a few educational points. ${ }^{48}$ This has proved very popular already and we have had numerous interesting submissions (table 1). The Images series is now available free of charge in a collection on the Thorax website (http://thorax. bmjjournals.com/cgi/collection/images). Our thanks to Mark Fitzgerald and his team of Nestor Muller and Jim Hogg in Vancouver for organising this section each month.

We would like to thank all our authors who have sent us such high quality papers for review. The number of letters to the editor submitted to the journal has also increased this year and we encourage submission of both letters commenting on published articles and also research letters describing original results that may be of a pilot nature. Please see the website for instructions concerning submissions of letters to Thorax. We would like to thank all the many reviewers who have given up their valuable time to assess papers for Thorax and contributed to the success of the journal. ${ }^{49}$ A list of all the reviewers for Thorax during the past 12 months is published after this editorial.

Our thanks go to all the Associate Editors who have provided invaluable help in selecting the best papers for publication and to the International Advisory Board for their support of the journal. During the year we held a meeting in November 2003 in London with all the Editors and Associate Editors and another meeting with the Thorax Advisory Board at the ATS 
Table 1 Submissions to Thorax by article type

\begin{tabular}{llllll}
\hline & $\mathbf{2 0 0 0}$ & $\mathbf{2 0 0 1}$ & $\mathbf{2 0 0 2}$ & $\mathbf{2 0 0 3}$ & $\mathbf{2 0 0 4}$ \\
\hline Original research & 505 & 573 & 592 & 860 & 1028 \\
$\quad$ Full papers & 34 & 36 & 44 & $\mathrm{~N} / \mathrm{A}$ & $\mathrm{N} / \mathrm{A}$ \\
$\quad$ Short papers & 6 & 9 & 6 & $\mathrm{~N} / \mathrm{A}$ & $\mathrm{N} / \mathrm{A}$ \\
$\quad$ Rapid communications & 165 & 186 & 146 & 211 & 285 \\
Case reports & 56 & 55 & 33 & 77 & 104 \\
Editorials/reviews & 14 & 15 & 40 & 26 & 12 \\
Review series & 46 & 5 & 16 & $\mathrm{~N} / \mathrm{A}$ & $\mathrm{N} / \mathrm{A}$ \\
Supplement articles & 96 & 95 & 70 & 82 & 99 \\
Letters & $\mathrm{N} / \mathrm{A}$ & $\mathrm{N} / \mathrm{A}$ & $\mathrm{N} / \mathrm{A}$ & 4 & 69 \\
Images in Thorax & 922 & 974 & 947 & 1260 & 1597 \\
Total & & & & &
\end{tabular}

Table 2 Geographical distribution of submissions

\begin{tabular}{lrrrrr}
\hline & $\mathbf{2 0 0 0}$ & $\mathbf{2 0 0 1}$ & $\mathbf{2 0 0 2}$ & $\mathbf{2 0 0 3}$ & $\mathbf{2 0 0 4}$ \\
\hline UK & 348 & 323 & 325 & 378 & 398 \\
Europe excluding UK & 310 & 386 & 329 & 437 & 527 \\
USA and Canada & 87 & 68 & 74 & 126 & 146 \\
Japan & 56 & 69 & 74 & 99 & 107 \\
Australasia & 60 & 57 & 45 & 67 & 86 \\
Asia & 39 & 26 & 41 & 85 & 125 \\
South America & 5 & 7 & 6 & 21 & 23 \\
Africa & 4 & 5 & 1 & 6 & 11 \\
Middle East & 13 & 24 & 21 & 21 & 26 \\
\hline
\end{tabular}

Meeting in Orlando, USA in May 2004. Ed Howard, our editorial assistant, has performed a superb job in running the journal and in June we were delighted that he was promoted to a new post in BMJ Publishing of Development Editor. Ed now has charge of a number of journals but still has an active role in the administration of Thorax. Julia Cresswell, who previously worked part time for the journal, is now our full time editorial assistant. We would like to thank Ed and Julia for all their hard work over the year to ensure that the journal runs smoothly and efficiently. We also thank Andrea Horgan, the managing editor, for all her support and advice and Liz Stockman, the technical editor, for ensuring that the monthly issues of Thorax are produced to the highest standard. Finally, we would like to thank Ed Neville, Sheila Edwards and the BTS for their support of Thorax over the year.

This has been a very busy year for the journal and, with the increase in high quality submissions, this has resulted in a relatively high rejection rate. We apologise to those authors who feel that we have not returned the appropriate decisions on their papers. However, with the increase in submissions this year we can assure you all a very successful journal in the coming year. The goal of our work as editors is to ensure that each month all Thorax readers can find something of interest to read in the journal.
Thorax 2004;59:1012-1015.

doi: 10.1136/thx.2004.036210

\section{Authors' affiliations}

J A Wedzicha, S L Johnston, D M Mitchell, Thorax Editorial Office, BMA House, London WCIH 9JR, UK

Correspondence to: Professor J A Wedzicha; i.a.wedzicha@qmul.ac.uk

\section{REFERENCES}

1 Wedzicha JA, Johnston SL, Mitchell DM. New Year, new editors. Thorax 2003;58:1-2.

2 Wedzicha JA, Johnston SL, Mitchell DM. Annual report 2003. Thorax 2003;58:1015-7.

3 Wedzicha JA, Johnston SL, Mitchell DM. Journal impact factors for 2003: Thorax increases. Thorax 2004;59:736.

4 Knox AJ, Britton J. Annual report October 2000 to September 2001. Thorax 2001;56:901.

5 Knox AJ, Britton J. We're off: Annual report October 2001 to September 2002. Thorax 2002;57:1003-4.

6 Tobin MJ. Thirty years of impact factor and the journal. Am J Respir Crit Care Med 2004;170:351-2.

7 Manhire A, Charig M, Clelland C, et al. Guidelines for radiologically guided lung biopsy. Thorax 2003;58:920-36.

8 Manhire AR, Richardson CM, Gleeson FV. Lung biopsy guidelines - for the obedience of fools and guidance of wise men. Thorax 2003;58:913-4

9 The National Collaborating Centre for Chronic Conditions. Chronic obstructive pulmonary disease: national clinical guideline on management of chronic obstructive pulmonary disease in adults in primary and secondary care. Thorax 2004:59(Suppl 1): 1-232.

10 Halpin D. NICE guidance for COPD. Thorax 2004;59:181-2.

11 Wedzicha JA. Peer review and NICE guidelines. Thorax 2004;59:183.
12 Sethi T. Lung cancer-Introduction. Thorax 2002;57:992-3.

13 Fong KM, Sekido Y, Gazdar AF, et al. Lung cancer-9: Molecular biology of lung cancer: clinical implications. Thorax 2003;58:892-900.

14 Wells FC. Lung cancer-10: Delivering a lung cancer service in the 21 st century. Thorax 2003;58:996-7.

15 Morice AH. Chronic cough-not such a heartsink. Thorax 2003;58:829.

16 Morice AH, Kastelik JA. Cough-1: Chronic cough in adults. Thorax 2003;58:901-7.

17 de Jongste JC, Shields MD. Cough -2 : Chronic cough in children. Thorax 2003;58:998-1003.

18 Fontana GA, Pistolesi M. Cough-3: Chronic cough and gastro-oesophageal reflux. Thorax 2003:58:1092-5.

19 Dicpinigaitis PV. Cough-4: Cough in asthma and eosinophilic bronchitis. Thorax 2004:59:71-2.

20 Morice AH, Geppetti P. Cough-5: The type 1 vanilloid receptor: a sensory receptor for cough Thorax 2004;59:257-8.

21 McGarvey LPA. Cough-6: Which investigations are most useful in the diagnosis of chronic cough? Thorax 2004;59:342-6.

22 Belvisi MG, Geppetti P. Cough-7: Current and future drugs for the treatment of chronic cough. Thorax 2004;59:438-40.

23 Fleetham J. Sleep disordered breathing awoken. Thorax 2004;59:5-6.

24 Stradling R, Davies RJO. Sleep-1: Obstructive sleep apnoea/hypopnoea syndrome: definitions, epidemiology, and natural history. Thorax 2004;59:73-8

25 Fogel RB, Malhotra A, White DP. Sleep-2: Pathophysiology of obstructive sleep apnoea/ hypopnoea syndrome. Thorax 2004;59:159-63.

26 Schlosshan D, Elliott MW. Sleep-3: Clinical presentation and diagnosis of the obstructive sleep apnoea hypopnoea syndrome. Thorax 2004;59:347-52.

27 Engleman HM, Douglas NJ. Sleep-4: Sleepiness, cognitive function, and quality of life in obstructive sleep apnoea/hypopnoea syndrome. Thorax 2004;59:618-22.

28 George CFP. Sleep-5: Driving and automobile crashes in patients with obstructive sleep apnoea/ hypopnoea syndrome. Thorax 2004;59:804-7.

29 Stoller JK. $\alpha_{1}$-Antitrypsin deficiency. Thorax 2004;59:92-3

30 Luisetti M, Seersholm N. $\alpha_{1}$-Antitrypsin deficiency-1: Epidemiology of $\alpha_{1}$-antitrypsin deficiency. Thorax 2004;59:164-9.

31 DeMeo DL, Silverman EK. $\alpha_{1}$-Antitrypsin deficiency-2: Genetic aspects of $\alpha_{1}$-antitrypsin deficiency: phenotypes and genetic modifiers of emphysema risk. Thorax 2004;59:259-64.

32 Needham M, Stockley RA. $\alpha_{1}$-Antitrypsin deficiency -3 : Clinical manifestations and natural history. Thorax 2004;59:441-5.

33 Lomas DA, Parfrey H. $\alpha_{1}$-Antitrypsin deficiency4: Molecular pathophysiology. Thorax 2004;59:529-35.

34 Stoller JK, Aboussouan LS. $\alpha_{1}$-Antitrypsin deficiency -5 : Intravenous augmentation therapy: current understanding. Thorax 2004;59:708-12.

35 Gibson GJ. Moran Campbell and clinical science. Thorax 2004;59:737-40.

36 Hurst JR, Choo-Kang BSW. A breath of fresh air? Report of the 2003 British Thoracic Society Winter Meeting. Thorax 2004;59: 190-3.

37 Wedzicha JA, Bhowmik A, Seemungal T. Call for Lung Alerts. Thorax 2003;58:193.

38 British Thoracic Society. British Thoracic Society guidelines for the management of suspected acute pulmonary embolism. Thorax 2003;58:470-83.

39 British Thoracic Society/Scottish Intercollegiate Guideline Network. British guidelines on management of asthma. Thorax 2003;58(Suppl I):i1-94.

40 British Thoracic Society. BTS guidelines for the management of community acquired pneumonia in adults. Thorax 2001;56:1-64

41 MacNee W, Calverley PMA. Chronic obstructive pulmonary disease-7: Management of COPD. Thorax 2003;58:261-5. 
42 Henry M, Arnold T, Harvey J. BTS guidelines for the management of spontaneous pneumothorax. Thorax 2003;58(Suppl II):ii39-52.

43 Maskell NA, Butland RJA. BTS guidelines for the investigation of a unilateral pleural effusion in adults. Thorax 2003;58(Suppl II):ii8-17.

44 British Thoracic Society. Non-invasive ventilation in acute respiratory failure. Thorax 2002;57:192-211.
45 White AJ, Gompertz S, Stockley RA Chronic obstructive pulmonary disease -6 The aetiology of exacerbations of chronic obstructive pulmonary disease. Thorax 2003:58:73-80.

46 West R, McNeill A, Raw M. Smoking cessation guidelines for health professionals: an update. Thorax 2000;55:987-99.
47 Davies CWH, Gleeson FV, Davies RJO. BTS guidelines for the management of pleural infection. Thorax 2003;58(Suppl II):ii18-28.

48 FitzGerald JM, Müller N, Hogg J. Images in Thorax. Thorax 2003:58:915.

49 Wedzicha JA, Johnston SL, Mitchell DM. Thank you to all Thorax reviewers. Thorax 2004:59:6-7.

REVIEWERS USED BETWEEN 1 OCTOBER 2003 AND 30 SEPTEMBER 2004

\begin{tabular}{|c|c|c|c|c|c|c|c|}
\hline R Aalbers & S Bell & A Chang & $\begin{array}{l}R \text { Dhand } \\
\text { A Diacon }\end{array}$ & R Garrod & S Holland & A Knox & $\begin{array}{l}\text { G Marks } \\
\text { R Marshall }\end{array}$ \\
\hline $\begin{array}{l}\text { S Aaron } \\
\text { I Adcock }\end{array}$ & R Benson & M Chan-Yeung & $\begin{array}{l}\text { A Diacon } \\
\text { G Diette }\end{array}$ & J Gattınonı & $\begin{array}{l}\text { Jolloway } \\
\text { J P Homasson }\end{array}$ & $\begin{array}{l}\text { L Kobzık } \\
\text { T Koga }\end{array}$ & $\begin{array}{l}\text { R Marshall } \\
\text { J Martin }\end{array}$ \\
\hline B Adel & M Berger & A Chaouat & W Dik & D Geddes & S Hooper & D Kohler & R Martin \\
\hline K Adler & D Bergmans & C Chaparro & P Dilworth & J Gerritsen & I Horvath & J Kolbe & F Martinez \\
\hline E Aertgeerts & S Bergoñón & A Chauhan & G Dimopoulos & J Gibson & M Hotopf & K Kostikas & N Maskell \\
\hline A Agusti & J-F Bernaudin & F Chen & R Dinwiddie & N Gibson & D Howell & H Kramer & J Massie \\
\hline T Ajithkumar & D Bernstein & $\mathrm{H}-\mathrm{H}$ Chen & M Divjak & P Gibson & S Howie & C Kroegel & P Matricardi \\
\hline M Akira & M Berridge & A Chetta & R Djukanovic & M Giralt & R Hubbard & N Krug & M Max \\
\hline F Alabi & W Biernacki & P Chhajed & H Dockrell & A Glanville & W Hubbard & N Kuenzli & R Maynard \\
\hline S Aldington & D Bilton & E Chilvers & G Doering & N Glasgow & D Hui & R Kuhlen & M McElroy \\
\hline N Alexis & S Birring & M Chilvers & M Dolovich & J Glassroth & J Hull & P Lackie & R McEvoy' \\
\hline S Allen & H Bisgaard & S Chinn & G Donaldson & F Gleeson & J Hunt & F Laghi & R McFadden Jr \\
\hline E Alton & P Bishop & S-H Cho & L Donnelly & J Glynn & J Hurst & C Lai & L McGarvey \\
\hline K Alving & L Bjermer & D Christiani & J Dorin & D Godden & S Hussain & F Lake & S McKenzie \\
\hline R Alý & P Black & F Chung & N Douglas & S Godfrey & C Infante-Rivard & L Lands & T McManus \\
\hline B Ameredes & C Blackburn & A Churg & J Douglass & M Goetz & A Innes & C Laroche & K McNeil \\
\hline R Amin & J Blakey & C Ciudad & I Doull & D Gold & $M$ loanas & J Larson & W McNicholas \\
\hline H Amital & F Blasi & N Clark & $R$ du Bois & D Goldblatt & A lonescu & D Laws & C McSharry \\
\hline D Anantham & K Bloch & E Clini & A Duarte & Roger Goldstein & R Irwin & W Lawson & A Mehta \\
\hline G Anderson & A Boehler & J Coghlan & C Duboucher & Ron Goldstein & D Israel-Biet & B Leahy & P Merkus \\
\hline S Andreas & W Boersma & S Cohen & R Dweik & F Gordo & J Ivanyi & $\mathrm{R}$ Lemanske Jr & T Mickleborough \\
\hline T Andrew & A Bohadana & R Coker & K Eastham & R Gosselink & A Jackson & W Lenney & J Miles \\
\hline G Anhalt & S Bonini & J Coleman & P Eastwood & A Greening & A Jaffe & T F Leung & A Millar \\
\hline N Anthonisen & G Borasio & D Collie & F Edenborough & C Griffiths & R Jagoe & P Levy & R Miller \\
\hline M Antonelli & G Bothamley & A Condliffe & A Edwards & M Griffiths & A James & G Lewith & G Mills \\
\hline G Antunes & J Bott & S Connellan & J Egan & T Griffiths & $\mathrm{H}$ Jansen & $\mathrm{N}$ Li & M Miravitlles \\
\hline B Antus & D Bouros & G Connett & N Eiser & K Grimwood & C Janson & R Light & E Mitchell \\
\hline R Aris & J Bousquet & M Connolly & S Elborn & D Groneberg & J Janssen & C H Lim & M Moffatt \\
\hline P Arkwright & R Boyton & A Coonar & M Elliott & N Gross & M Janssen-Heijnen & D W T Lim & P Monagle \\
\hline L Armstrong & P Bradding & D Cooper & $J$ Emerson & S Guerra & N Jarad & W S Lim & E Monso \\
\hline K Ashutosh & D Bradley & G Cooper & M Emura & C Guilleminault & D Jarvis & M Lipman & H Montgomery \\
\hline J Aspa & P Brand & P Cooper & $\mathrm{H}$ Engleman & T Gustafsson & P Jeffery & M Lipseft & P Montuschi \\
\hline L Atzori & C Braun-Fahrlander & J-F Cordier & M Ennis & T Haahtela & A Jeffrey & B Lipworth & Y Moodley \\
\hline M Aubier & R Breen & M Corey & P Enright & H Hakanarson & J Johansson & A Litonjua & J Moore \\
\hline P Aurora & C Brightling & C Corrigan & S Ericksson & H Halliday & A Johnson & H Lode & J Moore-Gillon \\
\hline A Awomoyi & J Brisman & P Corris & A Ernst & D Halpin & B Johnson & C-G Lofdahl & M Morgan \\
\hline N Ayas & J Britton & M Cosio & P Ernst & D Hansell & I Johnston & E Lombardi & A Morice \\
\hline J Ayres & R Brouillette & U Costabel & $M$ Errington & F Hargreave & S Johnson & K Lui & M Morrell \\
\hline W Bailey & J Brown & M Cotton & S Erzurum & M Harries & A Jones & M Luisetti & N Morrell \\
\hline A Baisi & K Brown & E Creutzberg & M Everard & M-A Harris & P Jones & S Lum & M Morris \\
\hline Y Balabanova & S Brown & A Crockett & M Extermann & B Harrison & G Joos & B Lundback & J Moxham \\
\hline A Baldi & B Brunekreef & A Croft & T Fahey & T Harrison & M Joyce-Brady & B Lundgren & M Muers \\
\hline D Baldwin & V Brusasco & A Crossan & L Fairclough & N Hart & M Judson & G Lungarella & D Murdoch \\
\hline I Balfour-Lynn & P S Burge & P Cullinan & A Faith & T Hartert & B Jung & E Lyn & A Musani \\
\hline M Ballmann & N Burrows & S Cunningham & R Farre & J Harvey & E Juniper & D Lynch & D Musher \\
\hline E Bancalari & A Bush & A Custovic & B Fauroux & J Haughney & M Kaji & F Macbeth & P Nafstad \\
\hline E Bandera & C Butler & R Dahl & B Feltis & C Haworth & N Kalsheker & S MacDonald & P Navalesi \\
\hline H Bandla & C Byrnes & S E Dahlén & D Fennell & A Hayward & F Kanat & G Macfarlane & B Nemery \\
\hline A Banerjee & M Cábana & G D'Antona & R Fergusson & L Heaney & H Kanazawa & J Macfarlane & A Newman-Taylor \\
\hline A Bankier & T Cagatay & J D'Armiento & M Ferrer & A Hedley & R Kanner & J MacFarlane & R Newson \\
\hline E Baraldi & P Calverley & M Davey & A Fine & J Heffner & J Kaprio & G MacGregor & T Nicolai \\
\hline C Barber & J Calvert & B Davidson & D Fishwick & R Hegele & J Kastelik & W MacNee & T Ninan \\
\hline A Barker & I Campbell & C Davidson & J-W Fitting & R Heinzer & F Kauffmann & S Madge & G Nixon \\
\hline R Barker & M Campbell & M Davie & D Fitzgerald & P Helms & U Keil & M J Mador & H Nogami \\
\hline F Barlesi & G Canny & P Davies & M Fitzpatrick & J Henderson & F Kelly & K Maghni & A Nomura \\
\hline N Barnes & J Cantor & R Davies & D Fleming & D Hendrick & M Keilly & H Magnussen & P Noone \\
\hline E Barreiro & M Carby & F Daxboeck & L Flores-Suarez & R Henning & A Kendrick & R Mahadeva & M O'Driscoll \\
\hline E Bar-Yishay & I Carey & F De Baets & A Fogarty & M Henry & K Kerr & R Mahajan & K O'Byrne \\
\hline E Bateman & K H Carlsen & J de Bruyne & H Folgering & M Hetzel & H Kerstjens & D Mahler & C O'Connor \\
\hline J Batenburg & K Carlsen & J De Gracia & B Fonseca & C Hewson & T Kettle & A Majeed & W Oddy \\
\hline B Battistini & A C Carvalho & $\mathrm{J}$ de Jongste & G Fontana & P Hiemstra & S Kharitonov & H Mäkelä & M O'Doherty \\
\hline S Baudouin & R Casaburi & R de Marco & L Forbes & T Higenbottam & J Kidney & P Mallia & D O'Donnell \\
\hline B Baughman & P Cassano & E Dean & J Foweraker & B Higgins & J Kiely & J-L Malo & S O'Hickey \\
\hline C Beardsmore & D Cataldo & M Decramer & A Frew & M Hill & J Kimoff & F Maltais & D Olivieri \\
\hline R Beasley & M Cazzola & R Dekhuijzen & D Galetta & N Hirani & G King & J Mancini & L Olson \\
\hline R Beck & N Cebi & A Denjean & N Galie & A Hislop & J Kirk & A Manhire & D Orenstein \\
\hline M Becklake & D Center & M Denton & C Gallagher & E Hnizdo & B Klug & D Mannino & I Orme \\
\hline M Beers & G Chalmers & S Desai & M Gappa & J Hogg & P Knekt & S Manzoor Kadri & P Ormerod \\
\hline D Befus & R Chambers & G Devereux & J Garcia-Aymerich & S Holgate & D Knight & E Marchand & R Orriols \\
\hline E Bel & D Chan & C Dezateux & L Garcia-Marcos & F Holguin & M Knowles & C Marcus & L Osman \\
\hline
\end{tabular}




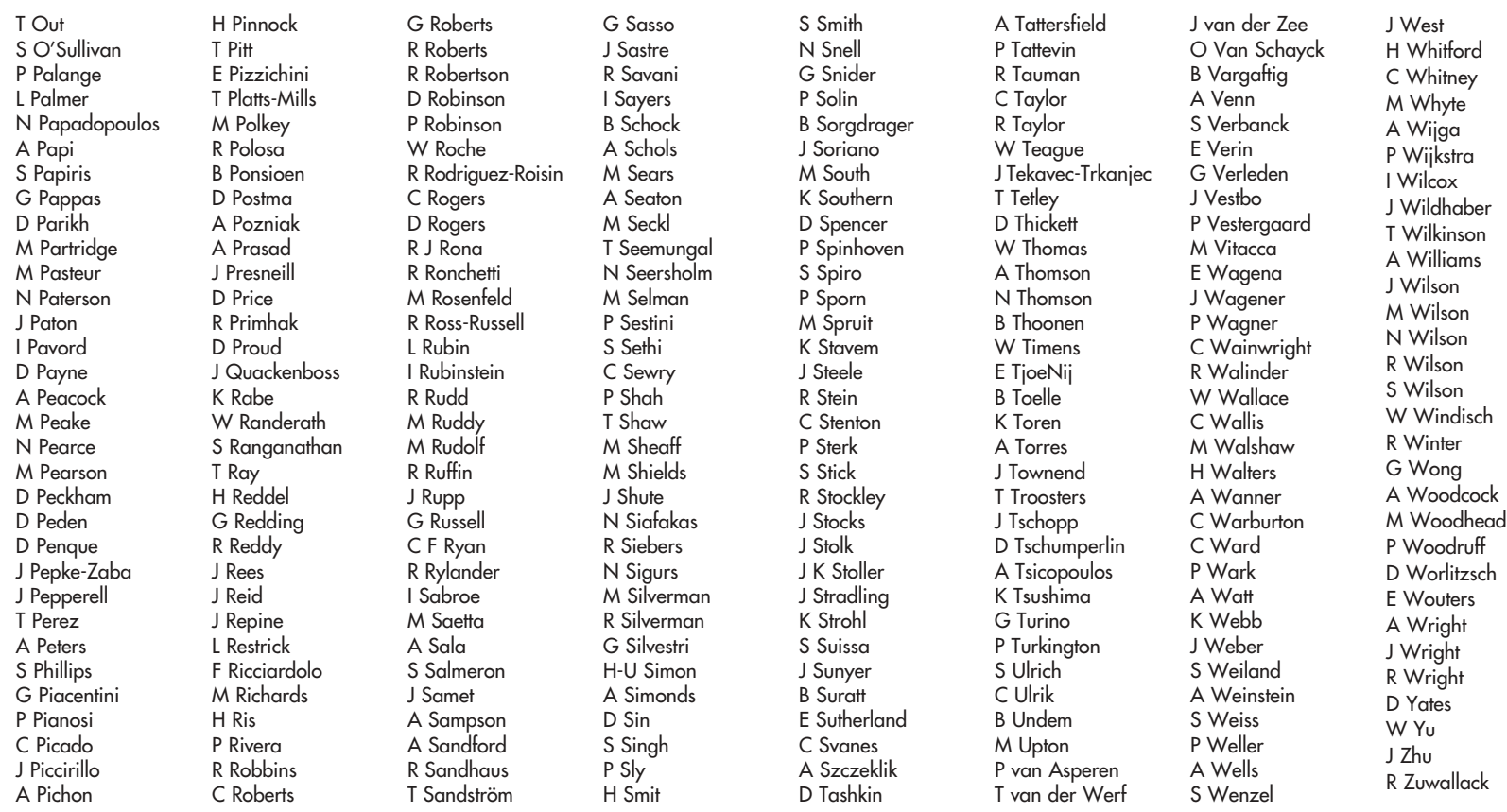

